\title{
A Method of Designing Interpretable Genetic Fuzzy Classification System Based On Mutating Parameters
}

\author{
Jl Hong ${ }^{a}$,Ma Ming ${ }^{b, *}$ \\ College of Information Technology and Media, Beihua University, Jilin 132013, China \\ aemail: jihongstudy@163.com, bemail:ma9063@163.com
}

Keywords: Fuzzy classification system; Word computing; expertise; mutating parameters

\begin{abstract}
This paper discusses the application of generating fuzzy rules with word computing in genetic fuzzy classification system, and proposes a new method to design genetic fuzzy classification system. The new algorithm generates initial fuzzy rules population with expertise of the randomly selecting samples, and adds mutating parameters to adjust the shape of membership function of fuzzy partition in order to expand the algorithm's search space. Experiments show that the new algorithm has better classification accuracy with shorter length of rules.
\end{abstract}

\section{Introduction}

The fuzzy sets which was proposed by professor Zadeh in 1965, has already been used widely in various application areas of the society[1][2]. Genetic fuzzy classification system is a hybridization of fuzzy logic and genetic algorithm, which was proposed in 1991, has become a hot spot in genetic algorithms[3][4][5][6]. There are two standards to judge whether a fuzzy classification system is good or not, accuracy and interpretability. Recently researches show that the two standards can not do best at the same time, it's better to find a compromise of them. Researchers proposed two approaches to solve it. One is generated fuzzy rules and the fuzzy rule sets by genetic algorithm to keep its accuracy first, and then promote its interpretability by some special methods, such as the similar simplify etc[7][8]. The other is generated both of them by humans' experiences to keep the interpretability first, and then promote its accuracy by genetic algorithm. Professor Zadeh[9] proposed to combine word computing to the second ways to adjust the shape of the membership function of fuzzy partition to promoting the accuracy while keep the interpretability. Li[10] chose four parameters represent "very", "little", "more or less", and "extreme" respectively. On the basis this paper proposes a new approach to design a fuzzy classification system, which generates initial fuzzy rules population with expertise of the randomly selecting samples, and adds mutating parameters to adjust the shape of the membership function of fuzzy partition during mutation process to expand search space of the algorithm to promote its efficiency. Experiments of wine data set show that the new approach has better classification accuracy and shorter rules length than the old ones.

The rest of this paper is organized as follows: Generate fuzzy partition with word computing is introduced in Section 2. The proposed algorithm is described in Section 3. The simulation and experimental results are presented in Section 4. Finally, concluding remarks are given in Section 5.

\section{Research of generate fuzzy partition with word computing}

Traditional fuzzy partition is split the whole search space into three or five parts with the same size. Although this fuzzy partition generation method is comprehensible, it has lower accuracy. Professor Zadeh considered that data information can connect with word computing by add language qualifiers to make the adjustment of fuzzy function equals to qualified language words, which made the fuzzy rules more interpretability. Li chose four parameters to adjust the shape of the function of every fuzzy partition, they are "very", "little”, "more or less", and "extreme”. Let us take wine data set for example to discuss the performance of the two methods. As we know wine data set has 13 attributes, if the algorithm uses five fuzzy partitions and “don't care” condition for 
each of them, the search space will be $(5+1)^{13}$, and if the word computing is added, it will grow to $(25+1)^{13}$. Adding word computing made the algorithm has better accuracy but lower convergence speed.

\section{A new design of genetic fuzzy classification system}

According to above analysis, this paper proposes a new approach which generates initial fuzzy rules population with expertise of the randomly selecting samples, and adds four mutating parameters to adjust the shape of membership function of fuzzy partition. The system design as follows:

1) Population initialization

This paper uses Triangular membership function to represents fuzzy sets, using real - coded, in which $(a, b, c)$ represents for the three vertex of the triangle.

$$
f(x ; a, b, c)=\max \left(0, \min \left(\frac{x-a}{b-a}, \frac{c-x}{c-b}\right)\right)
$$

First, split the whole search space of each dimension into five parts with the same size, then randomly select $M$ training samples from the training set, to each $x_{p i}(i \in[1, n])$, its compatibility $U_{i}$ with candidate membership function defined as

$$
U_{i}=\left|x_{p i}-b_{k, i}\right|
$$

Where $b_{k, i}$ is the second vertex of the five triangles. Then we got the expertise of this sample corresponding to the minimized $U_{i}$ of each dimension.

2) Consequent classes

To each rule $R_{j}$, its compatibility $u_{j}\left(x_{p}\right)$ with all training samples $x_{p}$ defined as

$$
u_{j}\left(x_{p}\right)=\prod_{i=1}^{\mathrm{n}} u_{j i}\left(x_{p}\right)
$$

To every consequent class, its $\beta_{\text {classh }}\left(R_{j}\right)$, sum of all training samples' $u_{j}\left(x_{p}\right)$ belongs to it defined as

$$
\beta_{\text {classh }}\left(R_{j}\right)=\sum_{x_{p} \in \text { classh }}\left(u_{j}\left(x_{p}\right)\right)
$$

We choose the class of $\max \beta_{\text {classh }}\left(R_{j}\right)$ as the consequent class of $R_{j}$. If more than two classes have same max $\beta_{\text {classh }}\left(R_{j}\right)$, we choose one with small quantity.

3) Fitness value

The fitness value fitness $\left(R_{\text {seti }}\right)$ is defined as

$$
\underset{i \in[1, L]}{\operatorname{fitness}}\left(R_{\text {seti }}\right)=\left\{\begin{array}{ll}
C P\left(R_{\text {seti }}\right)-\omega M P\left(R_{\text {seti }}\right), & M P\left(R_{\text {seti }}\right) \leq m \\
0, & M P\left(R_{\text {seti }}\right)>m
\end{array}\right\}
$$

Where $C P$ is the right classification and $M P$ is the wrong classification, $\omega$ is parameter of wrong classification punishment, which is belongs to [0.2, 0.5], $m$ is the max number of the wrong classification permitted.

4) Fuzzy genetic operations

Selection of this paper is combined roulette wheel selection strategy and elite selection strategy, and the mutation operation with mutating parameters as follows:

We select one rule from the rule set randomly, and choose two dimensions of it. To each dimension, change the first and the third vertex according to Eq.6 while keeping the second one unchanged.

$$
a_{j n_{1}}{ }^{\prime}=\max \left\{b_{j n_{1}}-\delta\left(b_{j n_{1}}-a_{j n_{1}}\right), x \min _{n_{1}}\right\} \quad c_{j n_{1}}{ }^{\prime}=\min \left\{b_{j n_{1}}+\delta\left(b_{j n_{1}}-a_{j n_{1}}\right), x \max _{n_{1}}\right\}
$$




\section{Simulation experiments}

Simulation results on wine data set under the same parameters are summarized in Table 1(with expertise) and Table 2(with out expertise).We choose 2/3 of it as training set, $1 / 3$ of it as testing set.

$\mathrm{Npop}=50, \mathrm{M}=6, \mathrm{Pc}=0.9, \mathrm{Pm}=0.1$. We choose four mutating parameters $0.4,0.7,1.3,1.6$.

Table 120 Experiments on wine data set with expertise

\begin{tabular}{|c|c|c|c|}
\hline Fuzzy partition & Training Set & Testing Set & Length of Rules \\
\hline $\begin{array}{c}\text { Five average fuzzy } \\
\text { partitions }\end{array}$ & $93.25 \%$ & $86.38 \%$ & 2.41 \\
\hline $\begin{array}{c}\text { Fuzzy partition of } \\
\text { word computing }\end{array}$ & $95.08 \%$ & $90.51 \%$ & 2.54 \\
\hline Our approach & $98.92 \%$ & $91.03 \%$ & 1.94 \\
\hline
\end{tabular}

Table 220 Experiments on wine data set with out expertise

\begin{tabular}{|c|c|c|c|}
\hline Fuzzy partition & Training Set & Testing Set & Length of Rules \\
\hline $\begin{array}{c}\text { Five average fuzzy } \\
\text { partitions }\end{array}$ & $86.67 \%$ & $80.00 \%$ & 2.8 \\
\hline $\begin{array}{c}\text { Fuzzy partition of } \\
\text { word computing }\end{array}$ & $94.33 \%$ & $88.79 \%$ & 2.45 \\
\hline Our approach & $93.75 \%$ & $87.93 \%$ & 1.75 \\
\hline
\end{tabular}

Through the simulation results in Table 1 and Table 2, our approach has higher classification accuracy and shorter rules length than the five average fuzzy partitions and the fuzzy partition of word computing.

\section{Conclusions}

The new method expands the search space while remaining system's interpretability, which is demonstrated to be more efficiency according to the simulation results. It also shows that adding expertise has good efficiency of the simulation results.

\section{Acknowledgments}

This work is supported by the Science and Technology Research Project of the Education Department of Jilin Province (No. 2012126) and Science and Technology Department of Jilin Province Natural Science Foundation（20140101185JC）

\section{Reference}

[1] Dong, J., Shen, G.J.: Remote Sensing Image Classification Based on Fuzzy Associative Classification. Journal of Computer Research and Development 49(7), 1500-1506 (2012)

[2] Chen, G., Feng, D.: Research on a new method for fuzzy rule weights in imbalanced data classification problem. Control and decision 27(1), 104-108 (2011)

[3] Huang, K.Q., Ren, W.J, Tan, T.N.: A Review on Image Object Classification and Detection. Chinese Journal of Computers 37(6), 1225-1240 (2014)

[4] Van de Sande K, Uijlings J, Snoek C, Smeulders A.: Hybrid coding for selective search. Proceedings of the workshop on PASCAL VOC. Florence, Italy, 1-8 (2012)

[5] Huang, K.Q., Tan T.N.: Review on Computational model for vision. Pattern Recognition and Artificial Intelligence 26(10), 951-958 (2013) 
[6] Bengio S.Weston J, Grangier D.: Label Embedding trees for large multi-class tasks. Proceeding of the Advances in Neural Information Processing Systems(NPIS), Vancouver, Canada, 163-171 (2010)

[7] Le Q V, Ranzato M A, Monga R, et al.: Building high-level features using large scale unsupervised learning. Proceedings of the international Conference on Machine Learning(ICML), Edinburgh, UK, 107-114 (2012):

[8] Li, J., Deng, Y.M., Shen, S.T.: Classification Rule Extraction Based on Fuzzy Area Distribution and Classification Reasoning Algorithm. Chinese Journal of Computers 31(6), 934-941 (2008)

[9] L. A. Zadeh.: Fuzzy logic = computing with words. IEEE Transactions on Fuzzy Systems, Vol.2, 103-111 (1996)

[10] Li, J.D., Zhang, X.J., Chen, Y.S.: Applying Expert Experience to Interpretable Fuzzy Classification Using Genetic Algorithms. Proceedings of the Fourth International Conference on Fuzzy Systems and Knowledge Discovery, Haikou, China, 129-133 (2007) 